\title{
Self-assembly behaviour of alkylpolyglucosides (APG) in mixed surfactant-stabilized emulsions system.
}

\begin{abstract}
The self-assembly behaviour of two alkyl polyglucosides (APG) surfactants; octyl/decyl polyglucosides (SAPG) and dodecyl/tetradecyl/hexadecyl polyglucosides (LAPG) in aqueous solution was studied. Critical micelle concentrations (CMC) of the surfactants were determined to predict onset point of surfactants self-assembly. The surfactants were prepared at concentrations $5 \times 10-3,5 \times 10-2$ and $5 \%(\mathrm{w} / \mathrm{w})$. Transmission electron microscopy (TEM) study showed that the surfactants with spherical-like structure self-assembled to simple rod-like, and then to aggregated rod-like structures with increasing concentration. The results showed that LAPG exhibited larger aggregate than SAPG. The distinctive evolution of self-assembly structures was observed when ethoxylated 3-(3-hydroxypropyl)heptamethyltrisiloxane (organosilicone) was incorporated into the APG system at 5\% (w/w) surfactant concentration. At mixed surfactant ratio (APG:organosilicone) of 9:1, TEM study showed that SAPG:organosilicone has multilamellar vesicles and LAPG:organosilicone has transient worm-like network. These mixed surfactants imparted difference in rheological behaviour, Newtonian and non-Newtonian, respectively, when they were developed into a microemulsion system of fatty acid methyl esters (FAMEs)/mixed surfactant/water. The dilution of the microemulsions with gentle stirring created a different geometry of nanoemulsions with oval and navette shapes for SAPG and LAPG, respectively.
\end{abstract}

Keyword: Self-assembly; Alkylpolyglucosides; Mixed surfactant; Microemulsions; Nanoemulsions. 